\section{Kopfschmerzprobleme bei Frauen}

\section{F. Bornatico-Valsangiacomo}

Die Prävalenz des weiblichen Geschlechtes bei Migräne (3:1) und Spannungskopfschmerzen (5:4) ist gut bekannt [1]. Während bei Spannungskopfschmerzen die Frauenprävalenz auf psychologische und psychosoziale Verhaltensunterschiede zurückzuführen ist [1, 2], gibt es bei Migräne Beobachtungen, die die Hypothese der hormonellen Einflüsse in der Schmerzentstehung unterstützen, nämlich der häufige Zusammenhang mit der Menstruation, die häufige Verbesserung während der Schwangerschaft, die Verschlechterung unter oralen Kontrazeptiva und während des Klimakteriums und die Besserung, die oft nach der Menopause eintritt.

\section{Menstruelle Migräne}

Die Bezeichnung "menstruelle Migräne» (MM) sollte für Migräneattacken reserviert werden, die ausschliesslich während der Zyklusblutung (1. Zyklustag \pm 2 Tage) auftreten [3]. Definitionsgemäss liegt eine reine periodenassoziierte MM lediglich bei weniger als $10 \%$ aller Migränikerinnen vor.

Klinisch sind die Attacken der MM durch hohe Schmerzintensität, lange Dauer und starke vegetative Begleitsymptome charakterisiert [4]. Öfters handelt es sich um Migräne ohne Aura.

Pathophysiologisch wird eine Reihe von theoretischen Mechanismen diskutiert. Davon erwähnenswert sind der Östrogenabfall und die Prostaglandinausschüttung aus dem Endometrium. Zum ersten hat Sommerville [5] nachgewiesen, dass die Verabreichung von Östradiol in der späten Lutealphase die erwartete Migräneattacke nachschiebt und die Zyklusblutung unverändert bleibt. Daraus die Hypothese, dass der plötzliche Abfall des langbestehenden hohen Östrogenspiegels für die Auslösung der Krise wahrscheinlich verantwortlich ist oder zumindest als Triggermechanismus anzusehen ist, der die Schwelle der Migräne senkt. Zum zweiten: Die Tatsache, dass die Prostaglandinausschüttung aus der Uterusschleimhaut ihre maximale Plasmakonzentration $24 \mathrm{~h}$ nach Beginn der Blutung erreicht, lässt die Vermutung zu, dass dieser Mechanismus bei der Entstehung jener Attacken wahrscheinlich eine Rolle spielt, die nach Beginn der Blutung auftreten [6].

Korrespondenz:

Dr. med. F. Bornatico-Valsangiacomo

Vice-Primario Servizio di Neurologia

Ospedale Civico

CH-6900 Lugano
Therapeutisch [4, 6, 7] sei zunächst betont, dass die MM schwieriger zu behandeln ist als die übliche Migräne, öfters therapieresistent ist und stark dazu neigt, nach vorübergehender Besserung wieder aufzutreten. Wichtig ist eine korrekte Diagnose, die durch einen entsprechenden Kalender zu erfassen ist. Dieser muss nicht zuletzt auch alle möglichen Triggerfaktoren dokumentieren, die die Migräneattacken auslösen können.

In einem ersten Schritt sollte man versuchen, wie bei der üblichen Migräne, Triggerfaktoren zu identifizieren und zu eliminieren (z. B.: zu wenig oder $\mathrm{zu}$ viel Schlaf, Stress, Angst, Sorgen, Traurigkeit, bestimmte Nahrungsmittel oder Nahrungskarenz, Alkohol).

Die medikamentöse Akuttherapie entspricht derjenigen der üblichen Migräne (Tab. 1). NSAR sind effektiv Prostaglandinhemmer und stellen Medikamente erster Wahl dar, insbesondere für die Attacken, die zwischen dem ersten und dritten Zyklustag beginnen. Triptane, die spezifischen Migränetherapeutika, sind auch für die MM sehr gut wirksam. Anhand von Studienresultaten haben insbesondere Sumatriptan und Zolmitriptan ihre Wirkung bei der MM bewiesen $[8,9]$. Ergotaminpräparate werden wegen der potentiellen Gefahr der Entstehung von chronischen Kopfschmerzen in der Akuttherapie immer weniger gebraucht. Insbesondere bei über $48 \mathrm{~h}$ andauern Krisen behalten sie allenfalls noch eine Indikation. Die Gesamtdosis von 4-5 mg pro Periode und pro Monat sollte nicht überschritten werden.

Ist die Akuttherapie ungenügend wirksam, kann eine medikamentöse Kurzzeitprophylaxe mit NSAR oder mit Östrogenen während 7-10 Tagen angewendet werden. (Tab. 1).

Wenn die Kurzprophylaxe nutzlos ist, besteht die Indikation für eine Standardprophylaxe (Betablocker, Ca-Antagonisten, Serotonin-Antagonisten, Valproat, Antidepressiva). Bei absoluter Therapieresistenz kann man den Östrogenantagonisten Tamoxifen oder andere hormonelle Manipulationen einsetzen, allerdings ist hier die Zusammenarbeit mit dem Gynäkologen oder dem Endokrinologen zu empfehlen. Bei absoluter Therapieresistenz sollte man schliesslich nicht zögern, die Richtigkeit der Diagnose zu überprüfen.

\section{Das prämenstruelle Syndrom}

Auch wenn Überlappungen bestehen, sollte man versuchen, die MM von Kopfschmerzen im Rahmen des prämenstruellen Syndroms zu unterscheiden. Typisch dabei ist das clusterartige Auftreten der Kopfschmerzen in der zweiten Zyklushälfte, assoziiert mit anderen charakteristischen Symptomen wie Müdigkeit, Reizbarkeit, Depression, Labilität, Appetitstörungen, Brustspannungen. Wichtig für die Diagnose des prämenstruellen Syndroms ist das Fehlen der ersten drei genannten fast obligaten Symptome während der ersten Zyklushälfte. Therapie [10]: viele Frauen sprechen auf einfache nicht-pharmakologische Stra- 


\section{Tabelle 1}

Therapie der menstruellen Migräne.

\begin{tabular}{|c|c|}
\hline Medikamenti & ttherapie \\
\hline 1. NSAR & $\begin{array}{l}\text { Paracetamol Brausetablette } 1000 \mathrm{mg} \\
\text { Ibuprofen Granulat } 600-1200 \mathrm{mg} \\
\text { Naproxen Suppositorium/Tablette } 500 \mathrm{mg} \\
\text { Acetylsalicylat Brausetablette/Beutel } 1000 \mathrm{mg}\end{array}$ \\
\hline Antiemetika & $\begin{array}{l}\text { Domperidon Brausegranulat } 20-60 \mathrm{mg} \\
\text { Metoclopramid Tablette/Suppositorium } 10-20 \mathrm{mg}\end{array}$ \\
\hline 2. Triptane & \\
\hline 3. Ergotamin & \\
\hline Kurzzeitprop & \\
\hline 1. NSAR & $\begin{array}{l}\text { Nimesulide } 2 \times 100 \mathrm{mg} 7-10 \text { Tage } \\
\text { Naproxen } 2 \times 500 \mathrm{mg} 7-10 \text { Tage }\end{array}$ \\
\hline 2. Östrogene & $\begin{array}{l}\text { rmal Gel/Patch } 50-100 \mathrm{ug} / \mathrm{Tag}, 7 \text { Tage } \\
\text { Estraderm } 2 \times / \text { Woche/ } 1 \text { Woche; Climara } 1 \times / 1 \text { Woche }\end{array}$ \\
\hline Standardprop & \\
\hline Wie bei der $i$ & Migräne, evtl. Dosiserhöhung perimenstruell. \\
\hline
\end{tabular}

\section{Tabelle 2}

Pharmakologische Therapie des prämenstruellen Syndroms.

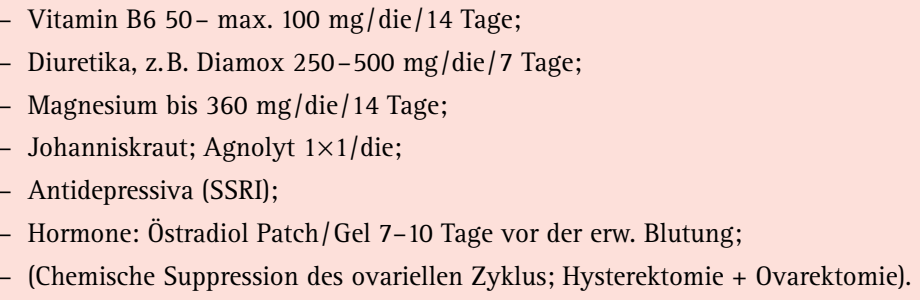

tegien an wie Entspannung, Yoga, Akupunktur. Manchmal können kohlenhydrathaltige Zwischenmahlzeiten helfen. Was die pharmakologische Therapie angeht, werden viele Medikamente empfohlen, wenige sind aber effektiv wirksam. (Tab. 2).

\section{Migräne und Schwangerschaft}

Mehr als 50\% der Migränikerinnen werden während des 2. und 3. Trimenons der Schwangerschaft beschwerdefrei, 25\% erfahren im 1. Trimenon eine Verschlechterung, 25\% bleiben unverändert. Die Besserung der Migräne soll auf dem gleichmässig hohen und stabilen Östrogenspiegel beruhen, die Verschlechterung post partum auf dessen Abfall [11]. Therapeutisch sind nicht-medikamentöse Massnahmen wie Schlaf, Entspannung, relaxierende Strategien oft ausreichend wirksam. Im Prinzip sind während der Schwangerschaft alle spezifischen Migränemittel (Triptane, Ergotaminpräparate, Serotoninagonisten) sowie auch NSAR und Metamizol kontraindiziert. Als einziges Medikament für die Akuttherapie ist Paracetamol erlaubt (Tab. 3). Acetylsalicylsäure ist zwischen dem 3. und 6. Monat erlaubt. Eine Migräneprophylaxe ist während der Schwan- gerschaft selten notwendig. Bei langdauernden und häufigen Attacken, die über den 1. Trimenon hinaus anhalten, können Beta-Blocker eingesetzt werden. Diese müssen spätestens 2 Wochen vor dem Termin abgesetzt werden. Alle anderen Migräneprophylaktika sind kontraindiziert $[11,12,15]$.

\section{Migräne und Antikonzeptiva}

Mehrere epidemiologische Studien zeigen, dass die Migräne v.a. in jüngeren Jahren mit einem leicht erhöhten Risiko für zerebrovaskuläre Insulte (CVI) verbunden ist. Mit 40 Jahren beträgt die relative Risikoerhöhung 2,8, mit 60 ist diese 1,7.

Diese Risikoerhöhung steigt signifikant $(6,8-8,6 \times)$ bei Migräne mit Aura im Vergleich zu Migräne ohne Aura. Studienergebnisse weisen darauf hin, dass Migräne mit Aura einen unabhängigen Risikofaktor für CVI darstellt. Weshalb die Migräne die Patientinnen zu CVI prädisponiert, ist nicht bekannt. Man vermutet, dass Veränderungen des Koagulationsprozesses, Veränderungen der Gefässwandkontraktilität und evtl. auch eine Thrombozytendysfunktion eine Rolle spielen können.

Aus der gemeinsamen Betrachtung des Risikos bei Migräne und Antikonzeptiva geht hervor, dass bei Migränikerinnen sowohl mit Aura wie auch ohne Aura und in jeder Altersklasse der Gebrauch von Antikonzeptiva die Inzidenz von CVI in etwa verdoppelt. Bei Vorhandensein weiterer Risikofaktoren für kardiound zerebrovaskuläre Erkrankungen wird das Risiko zusätzlich potenziert. Es ist z. B. bekannt, dass Migräne plus Antikonzeption und Rauchen mit einer hochsignifikanten Risikoerhöhung $(\times 30)$ verbunden ist.

In Mangel eines generellen Konsensus über die therapeutischen Konsequenzen seien hier einige praktische Empfehlungen wiedergegeben, die W. Becker anlässlich der "Women's Health Conference» (Toronto 1998) formulierte [13]:

- Frauen mit Migräne ohne Aura können Antikonzeptiva wahrscheinlich unbedenklich anwenden, sofern keine signifikanten Risikofaktoren vorhanden sind. Vorsicht ist geboten bei Frauen über 40 Jahre.

- Bei Frauen mit Migräne ohne Aura, die unter Antikonzeptiva eine signifikante Zunahme ihrer Migräne aufweisen, soll das Absetzen der Pille erwogen werden.

- Frauen mit sehr kurzer Aura (v.a. visueller Natur) haben eine relative Risikoerhöhung zu CVI, die eher für jüngere Patientinnen akzeptabel ist, aber nicht für andere (bei über 30j.). Hier sollte man das Risiko-Nutzen-Verhältnis abwägen und individuell entscheiden.

- Frauen mit langdauernden und komplexen Auren (z.B. Dysphasie, Hemiparesen) ist der Gebrauch von Antikonzeptiva streng abzuraten.

- Bei Frauen, die zum ersten Mal unter Antikonzeptiva eine Aura entwickeln, oder bei welchen vorbestehende Auren länger oder komplexer werden, muss die Pille abgesetzt werden. 


\section{Tabelle 3}

Migränetherapie in der Schwangerschaft.

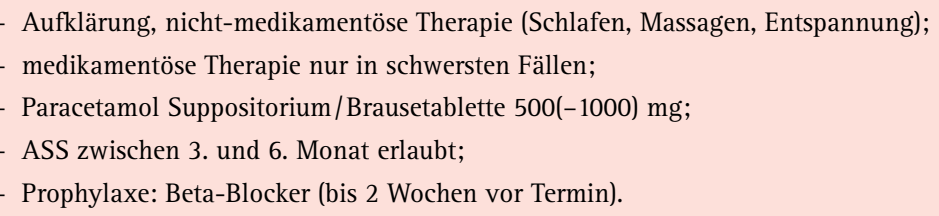

\section{Tabelle 4}

Migräne und Antikonzeptiva: Empfehlungen.

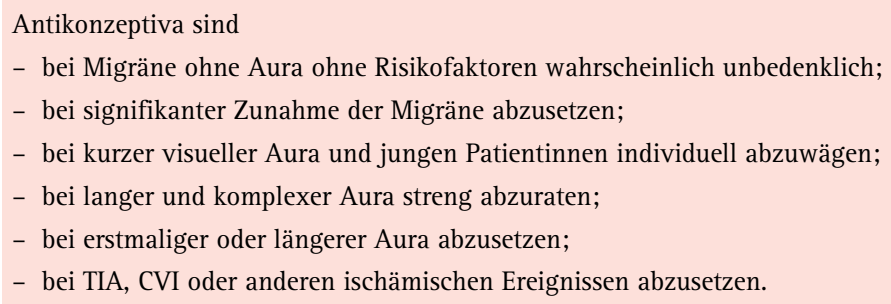

- In jedem Fall muss die Pille abgesetzt werden bei Frauen, die TIA jeglicher Art, CVI oder ischämische vaskuläre Ereignisse sonst erlitten haben.

Im weiteren ist zu ergänzen, dass v.a. bei Migränikerinnen für die Entscheidung Pille ja oder nein neben der Suche üblicher Risikofaktoren die zusätzliche Suche nach einer Thrombophylie bzw. einer Thromboseneigung inklusive Bestimmung der Homozysteins und der Antikardiolipin-Antikörper sinnvoll ist.

\section{Migräne in der Menopause}

Viele Frauen erfahren eine Exarzerbation ihrer Migräne vor und während der Menopause. Die Erklärung dafür wird in den starken und unregelmässigen Hormonfluktuationen während dieser Zeit gesucht. Diese Erklärung ist aber sicher ungenügend, da die Frauenprävalenz der Migräne auch spät nach der Menopause (2:1 bei $70 j$.) bestehen bleibt.

In der Menopause wird die kontinuierliche Substitutionstherapie mit kombinierten Hormonpräparaten immer populärer. Trotzdem beginnen viele Frauen in der Frühmenopause immer noch mit der zyklischen Substitution (Östrogene 3 Wochen/Monat). Diese Therapie kann bei den Frauen, die auf die Fluktuationen von Östrogen-Progesteron empfindlich sind, zu einer Exazerbation ihrer Migräne führen.

Therapeutisch [14]: Bei Östrogensubstitution kann eine Reduktion der Dosis nützlich sein. Als Alternative empfiehlt sich die Umstellung von der oralen auf die transdermale Verabreichung mit Patchs, die viel stabilere Hormonspiegel gewährleistet. Für Migränikerinnen bleibt jedoch die kontinuierliche Substitutionstherapie wahrscheinlich die vernünftigste Migräneprophylaxe.

Falls ungenügend wirksam, kann die Standardprophylaxe eingesetzt werden. Bei Gebrauch von Triptanen in der Akuttherapie ist besonderes in die- ser Altersklasse auf die Kontraindikationen (koronare Herzkrankheiten, CV-Erkrankungen, periphere Gefässkrankheiten und unkontrollierte arterielle Hypertonie) zu achten.

\section{Schlussfolgerungen}

Bis vor kurzem wurde den frauenspezifischen gesundheitlichen Aspekten wenig Beachtung geschenkt. Physiologische Unterschiede in der Funktion von Organen und Geweben zwischen Mann und Frau werden in den biologischen Wissenschaften zunehmend evident. Diese können klinische Krankheitsbilder und Verläufe verschiedenartig ausprägen. Die Migräne ist ein Beispiel dafür. Ihre Prävalenz, der Schweregrad und die Therapieempfindlichkeit werden von den verschiedenen endokrinologischen "Meilensteinen" des Frauenlebens beeinflusst. Einige klinische und therapeutische Aspekte der Migräne während der Menstruation, der Schwangerschaft und in der Menopause sowie bei Gebrauch von Antikonzeptiva werden im Artikel erörtert. Zusätzliche Kenntnisse über die pathophysiologischen Mechanismen des Verhältnisses zwischen Hormonfluktuationen und Migräne werden uns in einer vermutlich nicht allzu entfernten Zukunft erlauben, unsere Migränepatientinnen besser zu betreuen.

\section{Literatur}

1 Rasmussen BK, Jensen R, Schroll M, et al. Epidemiology of headache in a general population - a prevalence study. J Clin Epidemiol 1991;44:1147-57.

2 Rasmussen BK. Epidemiology of headache. Cephalalgia 1995; 15(1):45-68.

3 McGregor EA. "Menstrual" migraine: towards a definition. Cephalalgia 1996;16:11-21.

4 Diener HC. Menstruelle Migräne. In: Diener HC Kopf- und Gesichtsschmerzen. Stuttgart: Georg Thieme Verlag; 1997. S. 63-67.

5 Sommerville BW. The role of estradiol withdrawal in der etiology of menstrual migraine. Neurology 1972;22:355-9.

6 McGregor EA. Migraine and Menstruation. In: McGregor EA. Migraine in Women. London: Martin Dunitz; 1999. p. 32-46.

7 Schweiz. Kopfwehgesellschaft. Therapieempfehlungen für Kopf- und Gesichtsschmerzen. Schweiz Ärztezeitung 1995; 76(28):1180-4.

8 Salonen R, Saiers J. Sumatriptan is effective in the treatment of menstrual migraine: a review of prospective studies and retrospective analyses. Cephalalgia 1999;19:16-9.

9 Dalessio DJ, Brown DL, Solbach P, Adelman JU, Elkind AH, Stark SR. Oral 311C90 is effective in treatment of menstrual migraine. Cephalalgia 1996;16(5):400-1, Abs 112.

10 McGregor EA. Migraine and the premenstrual Syndrome. In: McGregor EA. Migraine in Woman. London: Martin Dunitz; 1999. p. 47-50.

11 Pfaffenrath V, Rehm M. Migraine in pregnancy: what are the safest treatment options? Drug Saf 1998;19(5):383-8.

12 Diener HC. Migräne und Schwangerschaft. In: Diener HC. Kopf- und Gesichtsschmerzen. Stuttgart: Georg Thieme Verlag; 1997. S. 65.

13 Becker WJ. Use of oral contraceptives in patients with migraine. Neurology 1999;53(Suppl 1):S19-S25.

14 Fettes I. Migraine in the menopause. Neurology 1999; 53(Suppl 1):S29-S33.

15 Aubé M. Migraine in Pregnancy. Neurology 1999;53(Suppl 1): S26-S28. 